\title{
FOCUSING OF GROUND VIBRATIONS GENERATED BY TRANS-RAYLEIGH TRAINS TRAVELLING WITH ACCELERATION
}

\author{
VV Krylov Loughborough University, Loughborough, Leicestershire, UK
}

\section{INTRODUCTION}

Over the last two decades, high-speed railways have been developed rapidly in many countries. The main reason for that is that they make a viable alternative to air transportation for short and medium distances, and they produce very low air pollution per passenger. However, as many other means of transportation, high-speed railways are not free of environmental problems. In particular, ground vibrations generated by high-speed railways is one of the major environmental problems that must be mitigated to allow high-speed trains to be used in densely populated areas ${ }^{1}$.

The intensity of railway-generated ground vibrations generally becomes larger at higher train speeds. For modern high-speed trains the increase in generated ground vibrations is especially large when train speeds approach certain critical velocities of elastic waves propagating in a trackground system. The most important of them is the velocity of Rayleigh surface wave in the supporting ground. As was theoretically predicted more than twenty years ago $^{2-4}$, if a train speed $v$ exceeds the Rayleigh wave velocity $c_{R}$ in supporting soil, the phenomenon of ground vibration boom occurs. This phenomenon is similar to a sonic boom for aircraft crossing the sound barrier, and it is usually associated with a very large increase in generated ground vibrations, as compared to the case of conventional trains. The existence of ground vibration boom has been later confirmed experimentally ${ }^{5,6}$. Therefore, it is now possible to speak of 'superseismic' or, more precisely, 'transRayleigh' trains ${ }^{7-9}$ in the same way as people speak of supersonic aircraft. The increased attention to the problems of ground vibrations associated with high-speed trains is reflected in a growing number of theoretical and experimental investigations in this area (see e.g. Refs. 1,10-14).

The essential difference between sonic boom and ground vibration boom is in the fact that, whereas sound velocity in the air is roughly the same in all locations above the earth surface, the Rayleigh wave velocity in the ground is different in different locations, depending on local geological properties of the ground. In some locations, where the ground is soft and marshy, Rayleigh wave velocities can be rather low, so that they can be exceeded by modern high-speed trains. For example, according to preliminary estimates based on geological data ${ }^{15}$, in some areas of the proposed HS2 high-speed railway network in the UK, the values of Rayleigh wave velocity can be rather low, around $80 \mathrm{~m} / \mathrm{s}$. Such velocities can be exceeded by operating HS2 trains that are expected to be travelling at speeds up to $400 \mathrm{~km} / \mathrm{h}$, i.e. $111.1 \mathrm{~m} / \mathrm{s}$. As a rule, train speeds even as high as $400 \mathrm{~km} / \mathrm{h}$ are still lower than Rayleigh wave velocities in the ground for the majority of locations. However, in the above-mentioned 'sensitive locations' characterised by low Rayleigh wave velocities, the phenomenon of ground vibration boom would represent a serious environmental problem to be reckoned with.

In the present paper, we will pay attention to the theory of one of the specific effects associated with the phenomenon of ground vibration boom, namely the effect of focusing of ground vibrations generated by high-speed trains. For a horizontally homogeneous ground, focusing can occur for trains travelling at trans-Rayleigh speeds, i.e. under the condition of ground vibration boom. Focusing under this condition occurs either for trains moving along a curved track or for trains travelling with acceleration, which is similar to focusing of a sonic boom from supersonic aircraft ${ }^{16-18}$, sometimes called a 'superboom'. In both these cases, i.e. trains moving along a curved track and 
trains travelling with acceleration, the magnitudes of generated ground vibrations increase in the areas of focusing. Some general results on this topic have been reported earlier by the present author ${ }^{19}$. In what follows, we will discuss the case of focusing of ground vibrations due to train acceleration in more detail. This type of focusing is more likely to happen in real practical situations as it takes place for straight tracks, where trains can travel at high speeds and with acceleration. The other type of focusing, due to track curvature, is less likely to happen in real situations because train speeds are always reduced on curved tracks to avoid loss of stability and train derailment.

\section{OUTLINE OF THE THEORY}

\subsection{Quasi-static Pressure Generation Mechanism}

In this sub-section, we provide some basic information that will be needed for understanding the phenomenon of focusing due to train acceleration. We remind the reader that the main mechanisms of railway-generated ground vibrations are: 1 ) the wheel-axle pressure onto the track; 2) the effects of joints in unwelded rails; and 3) the dynamically induced forces of carriage- and wheel-axle vibrations excited due to unevenness of wheels and rails. In what follows, we take into consideration only the first mentioned generation mechanism that is present even for ideally flat rails and wheels - namely, the quasi-static pressure of wheel axles onto the track, because this mechanism is also responsible for railway-generated ground vibration boom. The quasi-static pressure generation mechanism results from load forces applied to the railway track from each wheel axle that cause downward deflections of the track. These deflections move along the track at speed of the train, which results in a distribution of each axle load over all rail sleepers involved in the track deflection distance. Each sleeper, in turn, acts as a vertical force applied to the ground during the time necessary for a deflection curve to pass through the sleeper.

Being interested in fundamental features of the phenomenon of focusing of railway-generated Rayleigh waves, for the sake of simplicity, we will consider ground vibrations generated by a single axle load only. In the model under consideration, all properties of track and train, which determine generation of ground vibrations, are described by the function of load forces $P\left(t, x^{\prime}, y^{\prime}\right)$. We recall that for a single axle load moving at speed $v$ along a straight track (located at $y=0$ ), the time- and space-dependent load function has the form ${ }^{3,9}$ :

$$
P\left(t, x^{\prime}, y^{\prime}=0\right)=\sum_{m=-\infty}^{\infty} P\left(t-x^{\prime} / v\right) \delta\left(x^{\prime}-m d\right) \delta\left(y^{\prime}\right) .
$$

Here $P\left(t-x^{\prime} / v\right)$ is the time-delayed dynamic force acting from a sleeper with a coordinate $x^{\prime}$ to the ground surface, $d$ is the distance between neighbouring sleepers, and the delta-function $\delta\left(x^{\prime}-m d\right)$ takes the periodic distribution of sleepers into account. Using the explicit expression for $P(t)$ (see Refs. 4,8,9), taking the Fourier transform of (1) to calculate $P\left(x^{\prime}, y^{\prime}, \omega\right)$, and using the earlier developed theory8,9 involving the Green's function $G_{z z}(\rho, \omega)$, the following expression can be obtained for the vertical vibration velocity $v_{z}$ of Rayleigh waves generated on the ground surface $(z=0)$ at the point of observation with the coordinates $x, y$ by a single axle load moving along the straight track at speed $v$ :

$$
v_{z}(x, y, \omega)=P(\omega) D(\omega) \sum_{m=-\infty}^{\infty} \frac{1}{\sqrt{\rho_{m}}} \exp \left[i \frac{\omega}{v} m d+(i-\gamma) \frac{\omega}{c_{R}} \rho_{m}\right] .
$$

Here

Vol. 42. Pt. 1. 2020 


$$
D(\omega)=\frac{(-i \omega) q k_{R}^{1 / 2} k_{t}^{2}}{(2 \pi)^{1 / 2} \mu F^{\prime}\left(k_{R}\right)} \exp \left(-i \frac{3 \pi}{4}\right),
$$

where the factor $F^{\prime}\left(k_{R}\right)$ is a derivative of the so-called Rayleigh determinant

$$
F(k)=\left(2 k^{2}-k_{t}{ }^{2}\right)^{2}-4 k^{2}\left(k^{2}-k_{t}{ }^{2}\right)^{1 / 2}\left(k^{2}-k_{l}{ }^{2}\right)^{1 / 2}
$$

taken at $k=k_{R .}$. Other notations in equations (1)-(4) are as follows: $\rho_{m}=\left[(x-m d)^{2}+y^{2}\right]^{1 / 2}$ is the distance from the sleeper characterised by the number $m$ to the observation point located on the surface (with the coordinates $x, y$ ), $\omega$ is a circular frequency, $k_{R}=\omega / c_{R}$ is the wavenumber of a Rayleigh surface wave, $\quad c_{R}$ is the Rayleigh wave velocity, $k_{l}=\omega / c_{l}$ and $k_{t}=\omega / c_{t}$ are the wavenumbers of longitudinal and shear bulk elastic waves, where $c_{l}=\left[(\lambda+2 \mu) / \rho_{0}\right]^{1 / 2}$ and $c_{t}=$ $\left(\mu / \rho_{0}\right)^{1 / 2}$ are longitudinal and shear wave velocities, $\lambda$ and $\mu$ are the elastic Lame' constants, $\rho_{0}$ is mass density of the ground, $q=\left(k_{R}^{2}-k_{l}^{2}\right)^{1 / 2}$, and $\gamma=0.001-0.1$ is a non-dimensional loss factor describing the attenuation of Rayleigh waves in soil. The function $P(\omega)$ in equation (2) takes into account track dynamics (track flexural waves) and has the following form (see Refs. 4,8,9 for detail):

$$
P(\omega)=-\frac{12.8 \frac{T d}{v \pi^{2}}}{\frac{\omega^{4}}{\beta^{4} v^{4}}-4 \frac{\omega^{2}}{c_{\min }{ }^{2} \beta^{2}}-8 i \frac{g \omega}{c_{\min } \beta}+4},
$$

where $T$ is the axle load, $c_{\min }$ is the so-called minimal phase velocity of track flexural waves propagating in a track/ground system, $\beta$ is the parameter dependent on the elastic properties of track and ground and measured in $m^{-1}$, and $g$ is a non-dimensional track damping parameter. For relatively low train speeds, i.e., for $v \ll c_{\min }$, the full dynamic expression (5) for the force spectrum $P(\omega)$ goes over to the quasi-static one $e^{8,9}$.

A simple analysis of the expressions (1)-(5) shows ${ }^{3,4,7-9}$ that maximum radiation of ground vibrations takes place if the train speed $v$ is larger than Rayleigh wave velocity $C_{R}$. Under this condition, a ground vibration boom takes place, i.e., ground vibrations on the surface are generated as quasiplane Rayleigh surface waves symmetrically propagating at Mach angles $\Theta=\cos ^{-1}\left(c_{R} / v\right)$ in respect of the track. The amplitudes of generated ground vibrations in this case are much larger than in the case of conventional trains. It should be noted that for trans-Rayleigh trains these symmetrically propagating Rayleigh surface waves are generated equally well on tracks with and without railway sleepers, whereas for conventional trains the presence of sleepers for the possibility of generation of Rayleigh waves in the framework of quasi-static pressure generation mechanism is paramount ${ }^{3}$. As was mentioned above, the phenomenon of ground vibration boom is similar to the well-known phenomenon of sonic boom from supersonic aircraft.

In addition to the above-mentioned ground vibration boom, which is caused by train-generated Rayleigh waves propagating away from a railway track, another type of elastic waves, namely the so-called 'bow waves', i.e. track flexural waves propagating in the system track/ballast, can be also generated by high-speed trains if their speeds exceed the minimal phase velocity of track waves $c_{\min }$. The velocity $c_{\min }$ is indirectly related to Rayleigh wave velocity in the ground and is usually higher than Rayleigh wave velocity by $10-20 \%$. This means that for train speeds larger than $c_{\text {min }}$ the ground vibration boom and the bow wave phenomenon will take place simultaneously. The associated vertical dynamic displacements of the track in this case may exceed static displacements by 2-3 times, which would increase the magnitudes of generated ground vibrations also by 2-3 times and would adversely affect the train stability. In what follows, we will assume for

Vol. 42. Pt. 1. 2020 
simplicity that train speeds $v$, although exceeding Rayleigh wave velocity, are not high enough to exceed the minimal phase velocity of track waves

\subsection{Focusing of Generated Ground Vibrations due to Train Acceleration}

The effect of focusing of Rayleigh waves generated by high-speed trains travelling with constant acceleration can occur in the case of a train accelerating along a straight line if its current speed, $v$ $=v(t)=v_{0}+a t$, is higher than Rayleigh wave velocity in the ground $c_{R}$ (here $v_{0}$ is the initial train speed and $a$ is the acceleration) ${ }^{19}$. The geometrical acoustics interpretation of this effect is illustrated in Figure 1 for three different positions of a moving train characterised by the changed Mach angles of radiation of Rayleigh wave rays - angles $\theta_{1}, \theta_{2}$ and $\theta_{3}$ respectively. One can see that the caustic line (the area of focusing) in this case is not confined to the area near the train path, but is moving away from it as the train travels along the track and its speed increases.

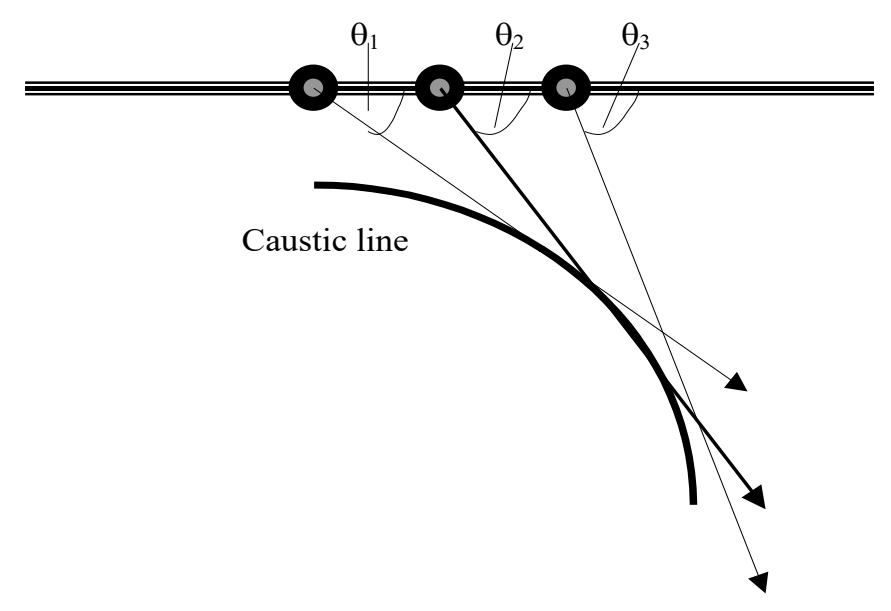

Figure 1. Geometrical acoustics interpretation of the focusing of Rayleigh waves radiated by a single axle load travelling with constant acceleration a along a straight track under the condition of ground vibration boom $\left(v>c_{R}\right)$. The focusing occurs along the caustic line that is moving away from the track ${ }^{19}$.

For quantitative analysis of this problem, it is convenient to express the train current speed $v$ as a function of the distance $s$ measured along the track. It follows from the kinematics of motion of a particle with acceleration $a$ that

$$
v=v_{0} \sqrt{1+\frac{2 a s}{v_{0}^{2}}} .
$$

The next step is to take into account the changing velocity $v$ described by equation (6) in equations (2) and (5). As can be seen from equations (2) and (5), the axle load velocity $v$ is present in the exponential factor of equation (2) and in the load function $P(\omega)$ given by equation (5). Expressing the distance along the track $s$ in equation (6) as $s=m d$ and substituting the resulting expression for $v$ into equations (2) and (5) gives the following formula for vertical component of the ground vibration velocity generated by a single axle load moving with acceleration ${ }^{19}$ : 


$$
v_{z}(x, y, \omega)=P(\omega) D(\omega) \sum_{m=-\infty}^{\infty} \frac{1}{\sqrt{\rho_{m}}} \exp \left[i \frac{\omega}{v_{0} \sqrt{1-2 a m d / v_{0}^{2}}} m d+(i-\gamma) \frac{\omega}{c_{R}} \rho_{m}\right]
$$

For shortness, the explicit expressions for $v$ in $P(\omega)$ are not shown in equation (7). The analysis shows though that the effect of changing velocity $v$ in the exponential factors of equations (2) and (7) on focusing of generated ground vibrations is dominant, so that changes of $v$ in $P(\omega)$ due to acceleration in many cases can be neglected and one can put $v=v_{0}$ in $P(\omega)$.

\section{NUMERICAL CALCULATIONS AND DISCUSSION}

The results of calculations of the spatial distribution of ground vibrations over the area of $50 \times 50 \mathrm{~m}$ generated at the frequency component $f=15 \mathrm{~Hz}$ by a single axle load travelling along the straight track with acceleration $a=1 \mathrm{~m} / \mathrm{s}^{2}$ are shown in Figure 2 in the form of a greyscale contour plot. The initial load speed was $v_{0}=82 \mathrm{~m} / \mathrm{s}$, and the velocity of Rayleigh wave in the ground was set as $c_{R}=80 \mathrm{~m} / \mathrm{s}$. Other relevant parameters were as follows: $d=0.7 \mathrm{~m}, \beta=1.28 \mathrm{~m}^{-1}$, and $\gamma=0.001$. It can be seen from Figure 2 that on both sides of the track the wave fronts of generated Rayleigh waves are formed symmetrically. However, focusing is hardly noticeable in this case of the initial train speed only slightly exceeding the Rayleigh wave velocity.

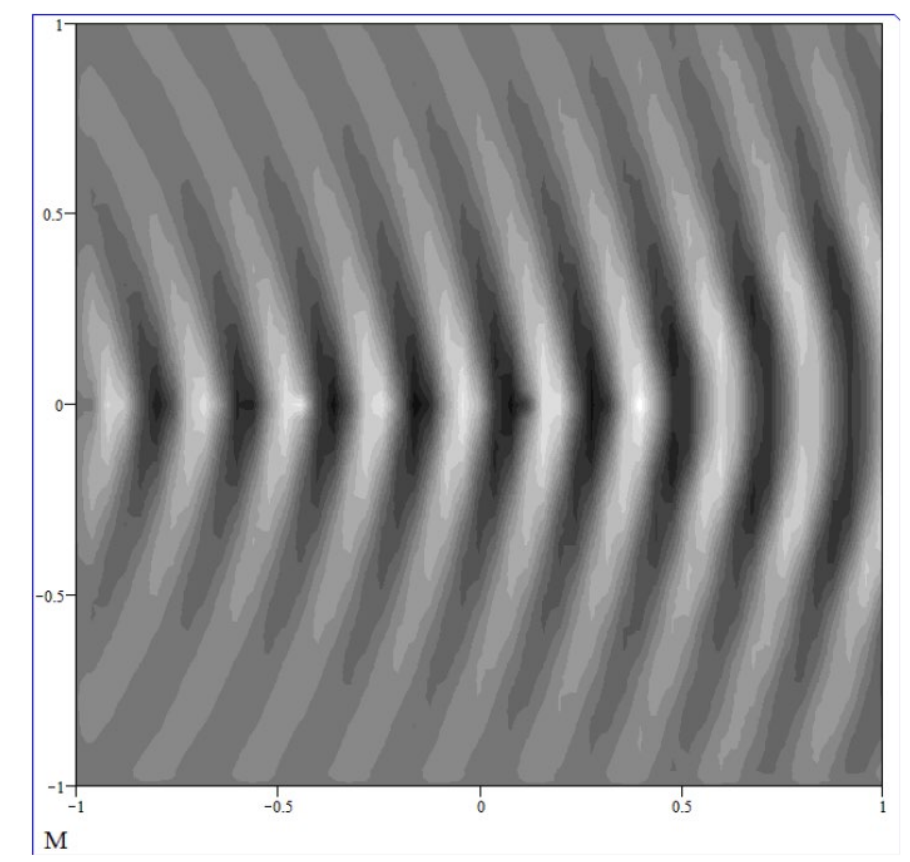

Figure 2. Spatial distribution of ground vibrations (in arbitrary units, greyscale contour plot) generated over the area of $50 \times 50 \mathrm{~m}$ by a single axle load travelling on a straight track with acceleration $a=1 \mathrm{~m} / \mathrm{s}^{2}$ and initial train speed $v_{0}=82 \mathrm{~m} / \mathrm{s}$.

Figure 3 shows the results of similar calculations for a single axle load travelling with the initial speed $v_{0}=90 \mathrm{~m} / \mathrm{s}$. Other parameters are the same as in Figure 2. It can be seen from Figure 3 that, in this case of higher initial train speed, the wave fronts of generated Rayleigh waves become 


\section{Proceedings of the Institute of Acoustics}

concave, and the focusing, which is associated with wave amplitudes amplification in the focusing areas, is clearly visible. This amplification is rather moderate, about two times, which is typical for this type of simple caustics, but it is added to the already large increase in ground vibration levels due to the presence of ground vibration boom. Note that the focusing areas (caustics) are moving away from the track with distance, in agreement with the geometrical acoustics interpretation discussed above.

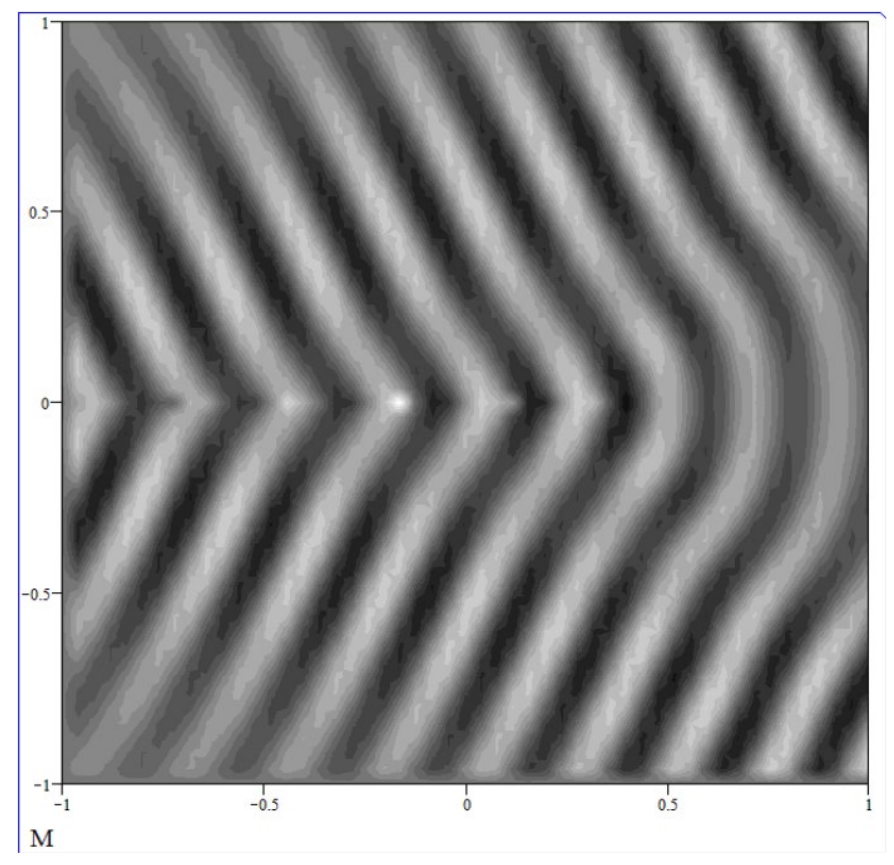

Figure 3. Spatial distribution of ground vibrations (in arbitrary units, greyscale contour plot) generated over the area of $50 \times 50 \mathrm{~m}$ by a single axle load travelling on a straight track with acceleration $a=1 \mathrm{~m} / \mathrm{s}^{2}$ and initial train speed $v_{0}=90 \mathrm{~m} / \mathrm{s}$.

It should be noted that the value of train acceleration $a=1 \mathrm{~m} / \mathrm{s}$ used in the above calculations is rather large. For example, the typical value of acceleration for French TGV trains is $a=0.255 \mathrm{~m} / \mathrm{s}^{2}$. It is applied when TGV trains depart from railway terminals and move until they reach the maximum operational speeds. A similar value of train acceleration is expected to be used for the proposed high-speed railway network HS2 in the UK. Calculations of spatial distributions of railway-generated ground vibrations for $a=0.255 \mathrm{~m} / \mathrm{s}^{2}$, which are not shown here for shortness, demonstrate that focusing is also present in this case, although it is less pronounced than in the case of $a=1 \mathrm{~m} / \mathrm{s}^{2}$ represented in Figure 3, as expected.

As was mentioned above, the preliminary estimates show that the proposed HS2 high-speed routes connecting London with Northern England via Birmingham at speeds up to $400 \mathrm{~km} / \mathrm{h}$ may give a possibility of ground vibration boom to occur in some sensitive locations characterised by low values of Rayleigh wave velocity (around $80 \mathrm{~m} / \mathrm{s})^{15}$. In order to reach the projected maximum operational speed $\left(v_{\max }=400 \mathrm{~km} / \mathrm{h}\right.$ for HS2), a train will have to pass a certain acceleration distance $s_{a}$. This distance can be easily expressed from Equation (6), in which one should put the initial velocity $v_{0}=0$ if a train departs from a railway terminal: 


$$
s_{a}=\frac{v_{\max }^{2}}{2 a} .
$$

Calculation for $a=0.255 \mathrm{~m} / \mathrm{s}^{2}$ and $V_{\max }=400 \mathrm{~km} / \mathrm{h}(111.1 \mathrm{~m} / \mathrm{s})$ gives the value of acceleration distance $s_{a}$ equal to $24.2 \mathrm{~km}$. This is a rather large distance, which illustrates the fact that in densely populated countries, with relatively short distances between railway terminals, a significant proportion of the routes, sometimes $40-60 \%$, may be covered by trains travelling with acceleration and deceleration ${ }^{15}$. This means that focusing of ground vibrations due to train acceleration in principle could be not so a rare occasion. However, one should not expect it to be frequently observed as railway operators try to avoid train operations at trans-Rayleigh speeds.

\section{CONCLUSIONS}

The effect of focusing of ground vibrations generated by high-speed trains travelling with acceleration along a straight line can occur if their current speeds are larger than Rayleigh wave velocities in the supporting ground. In this case, focusing of generated Rayleigh waves occurs symmetrically on both sides of the track, and it is accompanied by the corresponding increase in their amplitudes.

In densely populated countries, with relatively short distances between railway terminals, a significant proportion of the routes may be covered by trains travelling with acceleration and deceleration, which means that focusing of ground vibrations due to train acceleration could happen rather frequently in 'sensitive' locations along the proposed high-speed railway routes characterised by low Rayleigh wave velocities in the supporting ground. However, this does not mean that the described effect will be frequently observed in reality. The main reason for this is that railway operators try to avoid train operations at trans-Rayleigh speeds altogether. Nevertheless, it is important to understand that this effect does exist, and it should be taken into consideration, where appropriate, for more accurate predictions of ground vibration boom from high-speed trains.

\section{REFERENCES}

1. V.V. Krylov (ed), Ground Vibrations from High-speed Railways: Prediction and Mitigation, ICE Publishing, London (2019).

2. V.V. Krylov, On the theory of railway-induced ground vibrations, Journal de Physique IV, 4 (C5) 769-772 (1994).

3. V.V. Krylov, Generation of ground vibrations by superfast trains, Applied Acoustics, 44, 149164 (1995).

4. V.V. Krylov, Effect of track properties on ground vibrations generated by high-speed trains. Acustica-acta acustica, 84(1) 78-90 (1998).

5. C. Madshus and A.M. Kaynia, High speed railway lines on soft ground: dynamic behaviour at critical train speed, Proc. 6th International Workshop on Railway and Tracked Transit System Noise, 108-119. Ile des Embiez, France (1998).

6. K. Adolfsson, B. Andreasson, P.-E. Bengtsson and P. Zackrisson, High speed train X2000 on soft organic clay - measurements in Sweden, in F.B.J. Barends et al. (eds), Proceedings of the 12th European Conference on Soil Mechanics and Geotechnical Engineering, "Geotechnical Engineering for Transportation Infrastructure", Amsterdam, The Netherlands, 7-10 June 1999, Vol. 3, 1713-1718, A.A. Balkema, Rotterdam (1999).

7. V.V. Krylov, Ground vibration boom from high-speed trains, Journal of Low Frequency Noise, Vibration and Active Control, 18(4) 207-218 (1999).

8. V.V. Krylov, A.R. Dawson, M.E. Heelis and A.C. Collop, Rail movement and ground waves caused by high-speed trains approaching track-soil critical velocities, Proceedings of the 
Institution of Mechanical Engineers, Part F: Journal of Rail and Rapid Transit, 214, 107-116 (2000).

9. V.V. Krylov, Generation of ground vibration boom by high-speed trains, in V.V. Krylov (ed), Noise and Vibration from High-Speed Trains, 251-283, Thomas Telford Publishing, London (2001).

10. H. Takemiya, Ground vibrations alongside tracks induced by high-speed trains: prediction and mitigation, in: V.V. Krylov (ed), Noise and Vibration from High-Speed Trains, 347-393, Thomas Telford Publishing, London (2001).

11. A.V. Vostroukhov and A.V. Metrikine, Periodically supported beam on a visco-elastic layer as a model for dynamic analysis of a high-speed railway track, International Journal of Solids and Structures, 40(21) 5723-5752 (2003).

12. X. Sheng, C.J.C. Jones and D.J. Thompson, A theoretical study on the influence of the track on train-induced ground vibration, Journal of Sound and Vibration, 272, 909-936 (2004).

13. L. Auersch, The excitation of ground vibration by rail traffic: theory of vehicle-track-soil interaction and measurements on high-speed lines, Journal of Sound and Vibration, 284, 103-132 (2005).

14. G. Lombaert and G. Degrande, Ground-borne vibration due to static and dynamic axle loads of InterCity and high-speed trains, Journal of Sound and Vibration, 319, 1036-1066 (2009).

15. V.V. Krylov and B. Lewis, Assessment of locations along the proposed HS2 Routes that are likely to experience ground vibration boom from high-speed trains, Proceedings of the Institute of Acoustics, 38(1), 453-464 (2016).

16. D.J. Maglieri and K.J. Plotkin, Sonic boom, in Aeroacoustics of Flight Vehicles: Theory and Practice, Vol. 1: Noise Sources, 519-561, NASA, Langley Research Center (1991).

17. T. Auger and F. Coulouvrat, Numerical simulation of sonic boom focusing, AIAA Journal, 40(9) 1726-1734 (2002).

18. R. Blumrich, F. Coulouvrat and D. Heimann, Variability of focused sonic booms from accelerating supersonic aircraft in consideration of meteorological effects, Journal of the Acoustical Society of America, 118(2) 696-706 (2005).

19. V.V. Krylov, Focusing of ground vibrations generated by high-speed trains travelling at trans-Rayleigh speeds, Soil Dynamics and Earthquake Engineering, 100, 389-395 (2017).

20. K.F. Graff, Wave Motion in Elastic Solids, Clarendon Press, Oxford (1975).

21. S.V. Biryukov, Y.V. Gulyaev, V.V. Krylov and V.P. Plessky, Surface Acoustic Waves in Inhomogeneous Media, Springer-Verlag, Berlin (1995). 